Revue des patrimoines

$34 \mid 2018$

Lieux de pouvoirs. Architectures administratives dans la France contemporaine, 1945-2013. Avant/Après la décentralisation

\title{
Le bâtiment des archives départementales du Val- de-Marne : un équipement du Nouveau Créteil de l'agence Badani et Roux-Dorlut
}

The departmental archives building for the Val-de-Marne department at Creteil by the architectural agency of Badani and Roux-Dorlut

Franca Malservisi

\section{(2) OpenEdition}

1 Journals

Édition électronique

URL : http://journals.openedition.org/insitu/15699

DOI : 10.4000/insitu. 15699

ISSN : 1630-7305

Éditeur

Ministère de la culture

Référence électronique

Franca Malservisi, « Le bâtiment des archives départementales du Val-de-Marne : un équipement du Nouveau Créteil de l'agence Badani et Roux-Dorlut », In Situ [En ligne], 34 | 2018, mis en ligne le 17 juillet 2018, consulté le 03 mai 2019. URL : http://journals.openedition.org/insitu/15699 ; DOI : 10.4000/insitu. 15699

Ce document a été généré automatiquement le 3 mai 2019.

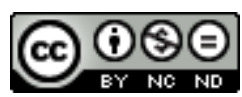

In Situ Revues des patrimoines est mis à disposition selon les termes de la licence Creative Commons Attribution - Pas d'Utilisation Commerciale - Pas de Modification 4.0 International. 


\section{Le bâtiment des archives} départementales du Val-de-Marne : un équipement du Nouveau Créteil de l'agence Badani et Roux-Dorlut

The departmental archives building for the Val-de-Marne department at Creteil by the architectural agency of Badani and Roux-Dorlut

\section{Franca Malservisi}

1 La réflexion sur le devenir de l'architecture du $\mathrm{xx}^{\mathrm{e}}$ siècle dépasse désormais le débat sur la patrimonialisation de ses réalisations emblématiques. Les équipements construits pendant les Trente Glorieuses ont contribué à façonner le paysage urbain en répondant à de nouvelles demandes et en affrontant de nouveaux défis. Comprendre les atouts et les fragilités des choix formels et constructifs retenus s'avère une étape indispensable pour assurer une gestion durable de ces édifices récents dont il nous faut parfois retrouver les clés de lecture. L'histoire de la conception, de la construction et de l'entretien du bâtiment des archives départementales du Val-de-Marne, à Créteil, conçu par les architectes Daniel Badani et Pierre Roux-Dorlut, offre un aperçu saisissant des enjeux multiples d'un nouvel équipement confronté aux aléas inhérents à l'expérimentation technique, à l'évolution des besoins et de la réglementation ainsi qu'à la transformation rapide et contraignante de l'environnement urbain (fig. 1). 
Figure 1

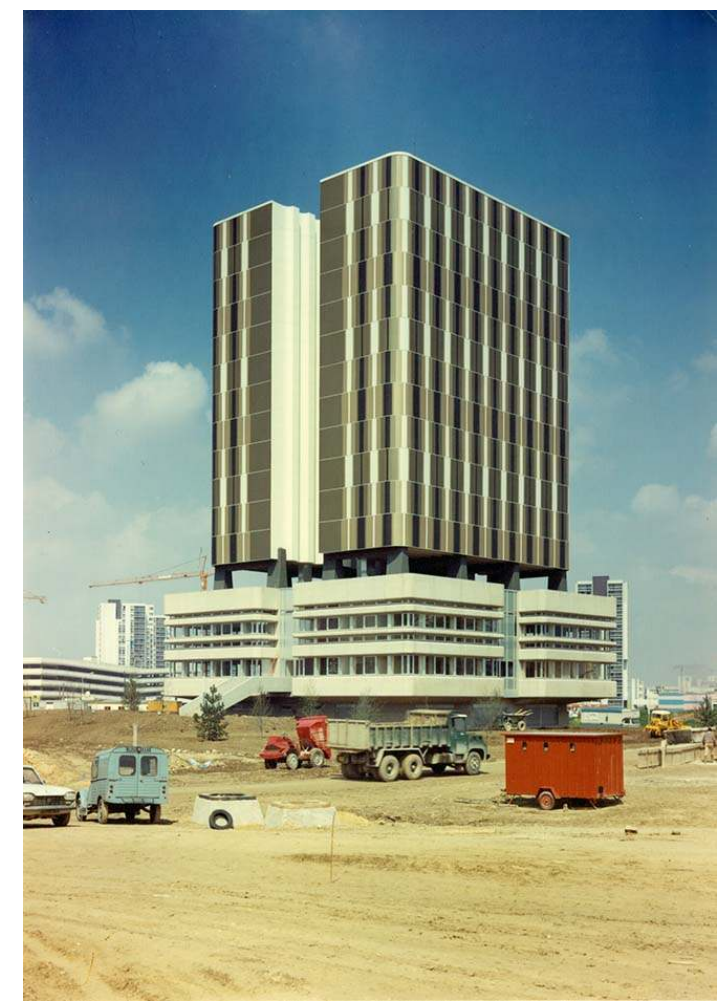

Archives départementales du Val-de-Marne : vue générale depuis le sud-ouest pendant l'aménagement des espaces extérieurs au cours de l'année 1974.

Phot. J. TH. Piek. (c) Archives départementales du Val-de-Marne, 45 W 53.

2 Le conseil départemental du Val-de-Marne poursuit aujourd'hui une démarche de mise en conformité et de réhabilitation $d u$ bâtiment. Dans le cadre de la mission d'accompagnement du CAUE du Val-de-Marne ${ }^{1}$, une étude associant diagnostic patrimonial et évaluation des questions énergétiques a été menée pour nourrir la réflexion sur les travaux à venir. Cet article reprend en grande partie le contenu de l'étude élaborée avec le soutien de la direction des archives départementales et de la direction des bâtiments².

\section{Le projet de bâtiment des archives départementales dans le Nouveau Créteil}

3 Un récent regain d'intérêt ${ }^{3}$ pour l'ensemble de l'œuvre des architectes Daniel Badani (1914-2006) et Pierre Roux-Dorlut (1919-1995) permet de situer le bâtiment des archives départementales du Val-de-Marne dans la vaste production de leur agence, fondée en $1946^{4}$. Il est cité dans le groupe de trois équipements presque contemporains: «les sculpturaux monuments qui ont fondé l'identité de la ville nouvelle de Créteil entre 1968 et [19]75: préfecture, palais de justice, archives $»^{5}$.

4 Historiquement, les locaux des archives départementales étaient liés aux préfectures, dont ils constituaient une annexe (Bourges, Brest, Limoges, Bar-le-Duc). Dans les années 1960, la construction de nouveaux équipements pour les archives départementales se fait souvent à la périphérie des villes et le lien avec la préfecture tend 
à se distendre. La singularité du projet de Créteil, comme de ceux réalisés pour les autres chefs-lieux de département suite à la restructuration de la région parisienne en 1964 (Nanterre, Hauts-de-Seine, et Bobigny, Seine-Saint-Denis), tient à l'insertion du projet dans un centre administratif nouveau, pour lequel le lien entre les archives départementales et la préfecture est pensé à l'échelle de la ville.

Devenue chef-lieu du nouveau département du Val-de-Marne en 1964, la commune de Créteil est transformée par la concrétisation d'un vaste projet urbain, le «Nouveau Créteil », projet ambitieux qui a mobilisé plusieurs urbanistes ${ }^{6}$. Après Michel Folliasson (1925-2011), en charge des premières propositions d'aménagement en 1962, le projet est revu et précisé par ses successeurs, Fernand Pouillon (1912-1986) puis Jean Fayeton (1908-1968). En 1968, au décès de ce dernier, le plan global est déjà bien avancé. Il prévoit un système de vastes ensembles immobiliers dont l'organisation doit répondre à une conception de la desserte favorisant la circulation automobile. Le territoire de la ZUP est découpé en quartiers de 600 à 1000 logements séparés entre eux par une voirie généreusement dimensionnée. Les plans de Fayeton intègrent déjà un centre structurant où sont prévus des équipements à échelle communale et départementale. Certains de ces équipements sont néanmoins prévus dans les autres quartiers pour contribuer à un équilibre général. Pierre Dufau (1908-1985), architecte en chef à partir de 1968, veille à la mise en œuvre de ce plan, structuré par un vaste lac artificiel et le réseau routier qui entoure les quartiers desservis par des parcours piétons. Un ensemble de passerelles relie un quartier à un autre en créant un système de circulations séparées qui complexifie l'organisation des entrées des immeubles et des équipements.

6 La réalisation de chaque quartier est confiée à un architecte qui définit les prescriptions pour des programmes réalisés ensuite par d'autres praticiens. L'architecte en chef Dufau garde la maitrise directe sur les quartiers du centre-ville et de L'Échat, où il prend en charge plusieurs réalisations, dont la plus emblématique est celle de l'hôtel de ville, surplombant le lac. Le choix des architectes ne passe donc pas par l'organisation de concours. Dufau et la Société anonyme d'économie mixte d'aménagement et d'équipement de la Ville de Créteil (SEMAEC), maître d'ouvrage délégué de la Ville, attribuent directement les programmes en faisant souvent appel à des architectes en chef des Bâtiments civils et Palais nationaux, comme c'est le cas pour Badani et Roux-Dorlut ${ }^{7}$.

7 Le paysage urbain de la première tranche du Nouveau Créteil est le résultat d'une organisation qui assure une liberté importante aux praticiens en répondant à la volonté d'éviter la monotonie, déjà très décriée, des grands ensembles. La juxtaposition d'expressions architecturales diverses est mise en scène à partir des ponts et passerelles piétonnes, véritables points de vue. Aménagé dans une vaste plaine, la nouvelle ville propose des jeux de hauteurs complexes entre les niveaux des circulations et les hauteurs des bâtiments. La figure de la tour ponctue le paysage urbain, en caractérisant le site, sans que la compréhension des hiérarchies entre les volumes et le repérage dans la ville en soient facilités. Des tours de logements se démarquent par leur hauteur et l'originalité $\mathrm{du}$ traitement de la façade en brouillant l'identification des équipements. La figure des tours n'est pas seulement liée à la volonté de façonner un nouveau paysage, elle permet également de répondre à la nécessité de rentabiliser des fondations onéreuses imposées par les caractéristiques des sols.

8 Les archives départementales du Val-de-Marne conservent un plan masse de 1968 qui donne un aperçu de l'organisation imaginée pour le secteur nommé initialement "quartier des Archives », devenu l'actuel quartier de la Croix-des-Mèches. Le nouvel 
équipement départemental avait été prévu à un emplacement relativement excentré, en application du principe de dissémination dans la ville d'une partie des équipements d'échelle départementale. Au cours de la définition de la première tranche du projet urbain, le bâtiment est finalement localisé au sud du quartier de la Brèche, avec un positionnement plus central. Dans le plan de la première tranche, datant de 1971, le bâtiment est déplacé à l'emplacement actuel et directement relié aux parcours piétons du « centre-ville » par des passerelles qui devaient enjamber l'avenue du Général-de-Gaulle et l'avenue de la Brèche. Le plan masse du premier projet présenté par les architectes Badani et Roux-Dorlut montre l'étendue et la complexité spatiale des parcours piétons en hauteur et des dalles prévues (fig. 2).

Figure 2

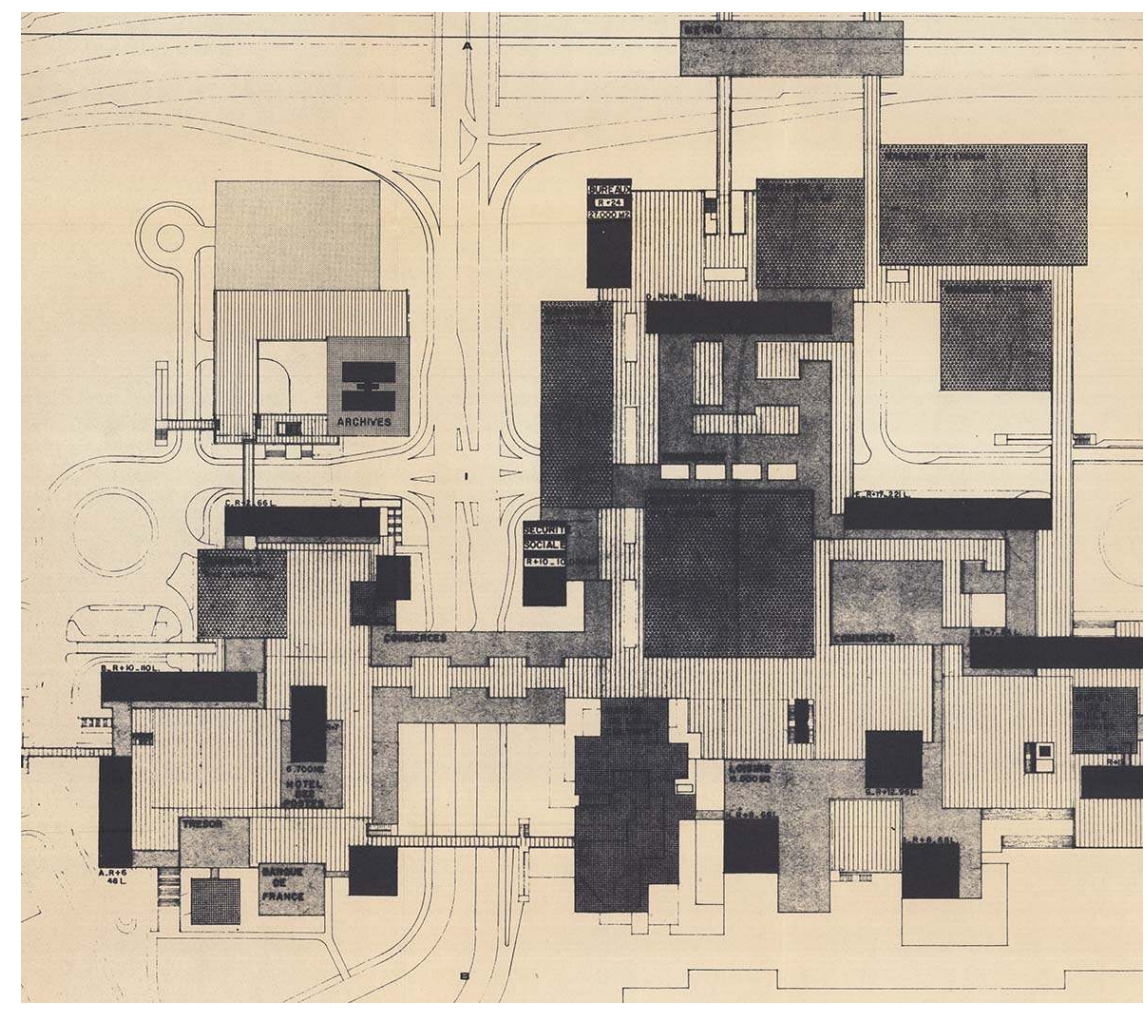

Détail du plan de situation du premier avant-projet présenté par les architectes Badani et Roux-Dorlut en 1969, éch. 1/1000. Les surfaces hachurées représentent les dalles et passerelles piétonnes dont le développement a été ensuite considérablement réduit.

(c) Archives départementales du Val-de-Marne, 1525 W 27.

Sans entrer dans le détail de l'évolution des conceptions urbaines, il faut rappeler que le projet d'aménagement subit des modifications considérables. La superficie du niveau haut, destiné à des espaces piétons animés par des commerces et des services, est considérablement réduite au cours de la réalisation de la première tranche. Le plan masse de la deuxième tranche, dont l'étude commence à partir du milieu des années 1970, entérine l'abandon de cette conception. Cette forte rupture entre les deux phases est accentuée par les retards liés aux crises économique et immobilière. L'évolution rapide des conceptions d'aménagement génère des raccords difficiles aux marges d'îlots aux morphologies trop diverses. Le bâtiment des archives se trouve à la lisière du centre-ville, dans un quartier (quartier de la Brèche) où l'organisation des déplacements et les programmes vont être sensiblement modifiés. Le bâtiment en garde les traces dans sa 
conception et sa réalisation, notamment dans les dispositions de l'entrée principale et l'aménagement des espaces extérieurs de la parcelle. En effet, entre 1970 et 1975, le réseau viaire du quartier de la Brèche est redessiné en relation avec l'évolution du programme prévu autour du site des archives et de la création du centre commercial d'échelle régionale. Au cours de ces errements du projet urbain, la voie longeant à l'ouest la parcelle du bâtiment des archives et la connexion en hauteur au réseau des circulations piétonnes sont abandonnées et le bâti doit s'adapter, tant bien que mal, au nouveau fonctionnement urbain.

\section{Évolution du projet}

Les documents conservés dans les fonds des archives départementales permettent de retracer l'historique du projet, depuis l'avant-projet de 1969 au bâtiment inauguré en 1975. Le parti général, qui superpose les deux volumes de magasins aux espaces de fonctionnement, reste inchangé depuis la première proposition de 1969. Les architectes apportent néanmoins des modifications non négligeables à chaque nouvelle phase d'évolution du projet.

Figure 3

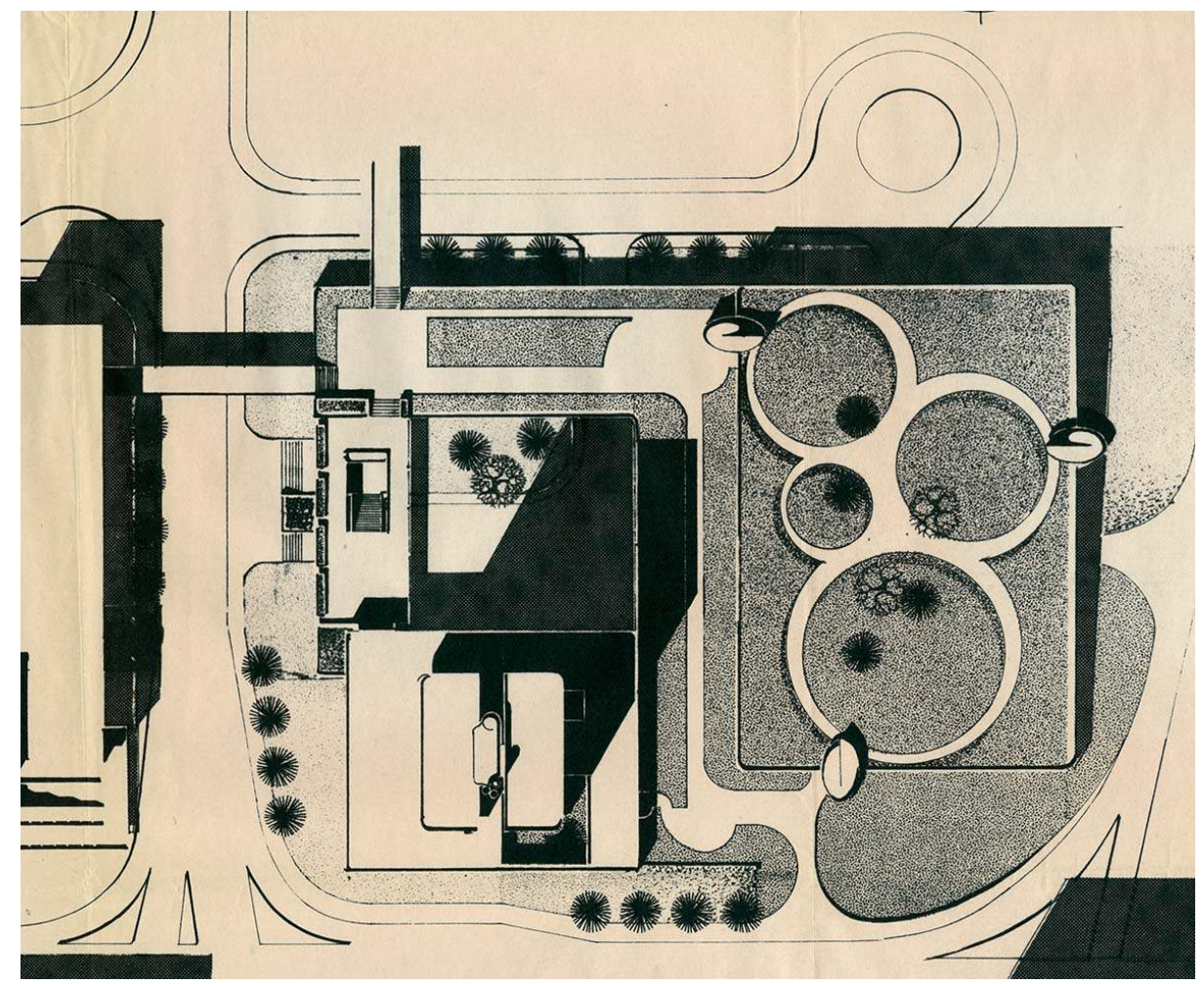

«Plan de masse d'ensemble », avant-projet 1969, détail. L'aménagement de la parcelle prévoit un vaste parking, couvert par une terrasse plantée, et des passerelles qui relient l'entrée de l'équipement au réseau des parcours piétons en hauteur. Avant-projet 1969, éch. 1/500.

(c) Archives départementales du Val-de-Marne, 1525 W 27.

11 En 1969, un premier avant-projet, établi en accord avec la direction générale des Archives, est présenté au conseil général des Bâtiments de France (fig. 3). Le procèsverbal de la séance du 29 mai 1969 décrit un bâtiment qui s'élève au centre d'un terrain 
de $7000 \mathrm{~m}^{2}$ et auquel on accède par une dalle piétonne située à $5 \mathrm{~m}$ au-dessus du terrain naturel. Un volume bas, à l'horizontalité très marquée, est affecté aux locaux administratifs, aux salles d'accueil du public et au logement de fonction. Au-dessus émergent les deux parallélépipèdes dédiés aux archives. La séparation en deux volumes divisés par une faille, où se trouvent les circulations verticales, permet de ne pas dépasser la surface maximale de stockage (fig. 4). Une dalle abritant les parkings est située à l'est du bâtiment. Le parti architectural prévoit « un double contraste pour les façades. L'un brutal qui oppose le volume fermé du stockage au pan de verre du R.D.C. haut. L'autre subtil: jeu de cylindres qui se dessine à l'intérieur de la fente existant entre les 2 bâtiments de stockage $»^{8}$.

Figure 4

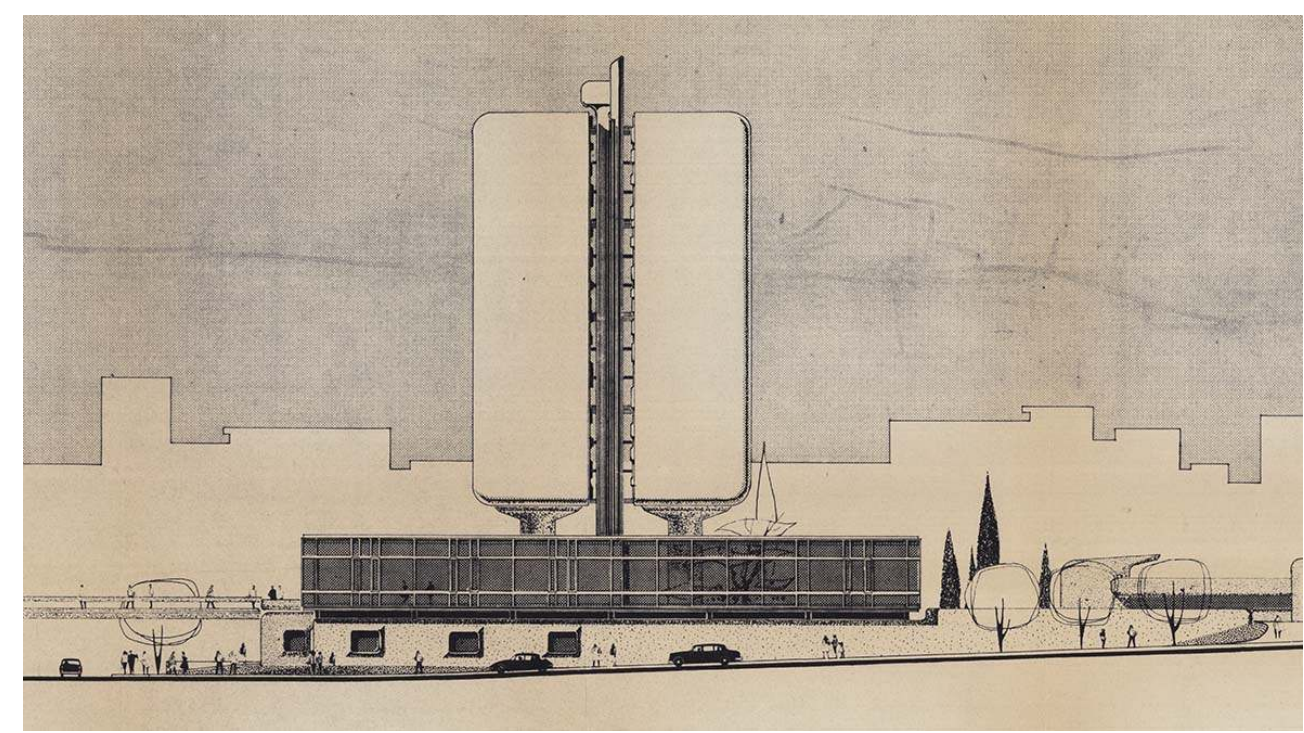

"Élévation sud », avant-projet de 1969, détail. Les circulations verticales qui desservent les deux silos des réserves sont situées au centre de la faille. Les angles arrondis des volumes des silos contrastent avec les arêtes vives du volume bas vitré. Avant-projet 1969, éch. 1/200.

(c) Archives départementales du Val-de-Marne, 1525 W 27.

Le rapporteur approuve ce projet défini comme « original ». Un des aspects marquants du projet est la mise en scène des silos par un traitement spécifique de l'enveloppe extérieure aveugle. Les architectes précisent que «l'habillage de la tour de stockage sera soit en métal soit en céramique $»^{9}$. Le choix de créer des silos pour abriter les magasins est lié à une évolution plus générale, celle des bâtiments d'archives entre les années 1950 et $1970^{10}$. La commission émet un avis favorable et demande d'étudier une réalisation en plusieurs tranches en raison du coût élevé des travaux, le montant de l'opération étant estimé à 12092650 francs. 
Figure 5

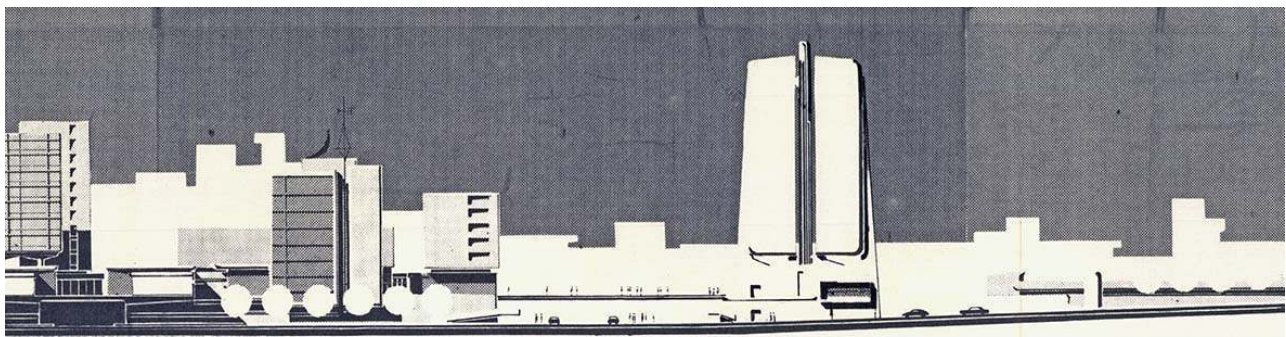

«Façades générales : sud et ouest », avant-projet 1970, détail de la façade sud. La passerelle donne toujours accès à l'espace d'accueil du public. Le volume des silos émerge parmi les autres constructions posées sur la continuité horizontale des dalles. Avant-projet 1970, éch. 1/200

(c) Archives départementales du Val-de-Marne, 1525 W 27.

13 Un deuxième avant-projet est proposé en mai 1970 (fig. 5). Dans le texte de présentation, les architectes justifient l'évolution du parti architectural :

Le résultat de la campagne de sondage qui fait ressortir des caractéristiques mécaniques du sol particulièrement mauvaises a orienté l'étude vers l'intégration de l'ensemble du programme dans un seul volume afin de limiter le nombre des puits de fondation nécessairement descendus à 40 mètres de profondeur ${ }^{11}$.

14 Le projet est alors estimé à 9432649 francs. Cette modification du fonctionnement structurel aboutit à une nouvelle proposition formelle. Les silos ne sont plus posés sur des piliers en retrait par rapport aux façades mais soutenus par quatre voiles formant d'énormes d'agrafes de blocage. Leurs bords évasés préfigurent les solutions plastiques adoptées au palais de justice que l'agence Badani et Roux-Dorlut réalise à Créteil entre 1975 et 1978 (fig. 6). Ce deuxième avant-projet prévoit toujours un accès au site en hauteur depuis l'ouest. Les documents graphiques montrent une entrée piétonne reliée à deux passerelles perpendiculaires qui enjambent les voies situées à l'ouest. 
Figure 6

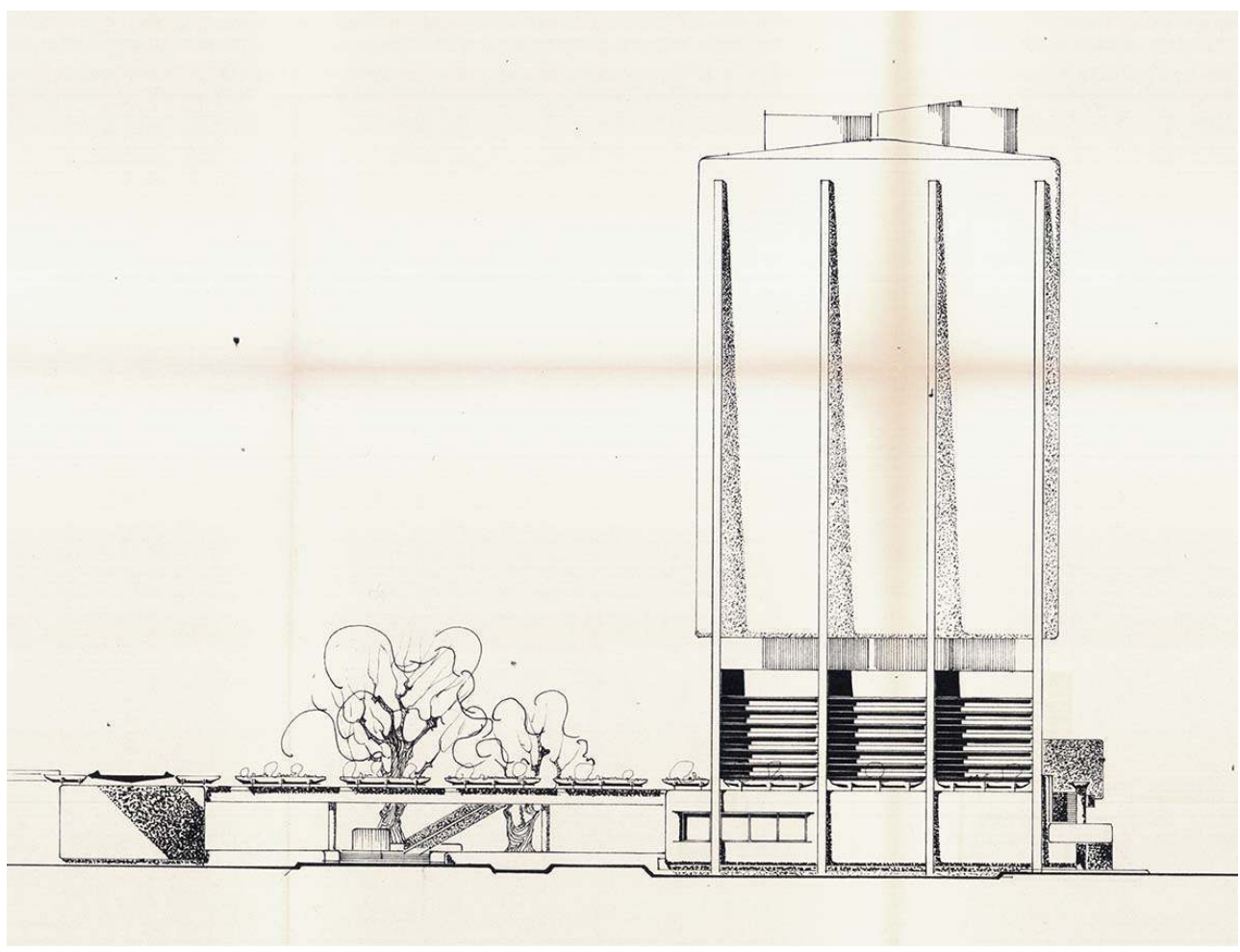

"Élévation sud », avant-projet de 1970, détail. Les voiles porteurs évasés relient les volumes hauts au soubassement en modifiant la logique du premier avant-projet. Le volume bas a été réduit et ne dépasse pas l'emprise des silos en raison des coûts induits par les caractéristiques du sol. Avantprojet 1970, éch. 1/200.

(c) Archives départementales du Val-de-Marne, 1525 W 27.

Le troisième projet, établi en 1971, est celui du dossier d'appel d'offres. L'aménagement des espaces extérieurs prend en compte la suppression du niveau de desserte piétonne en hauteur. Dans le plan masse, la voie située à l'ouest de la parcelle est devenue une voie en impasse, première étape d'un enclavement progressif du quartier de la Brèche. L'accès à l'équipement, isolé au centre d'une parcelle paysagée, se fait depuis cette voie en impasse. Malgré l'abandon des parcours piétons en hauteur, l'organisation des étages reste inchangée, ce qui impose la mise en œuvre d'une série d'escaliers extérieurs pour accéder à l'entrée (fig. 7). 


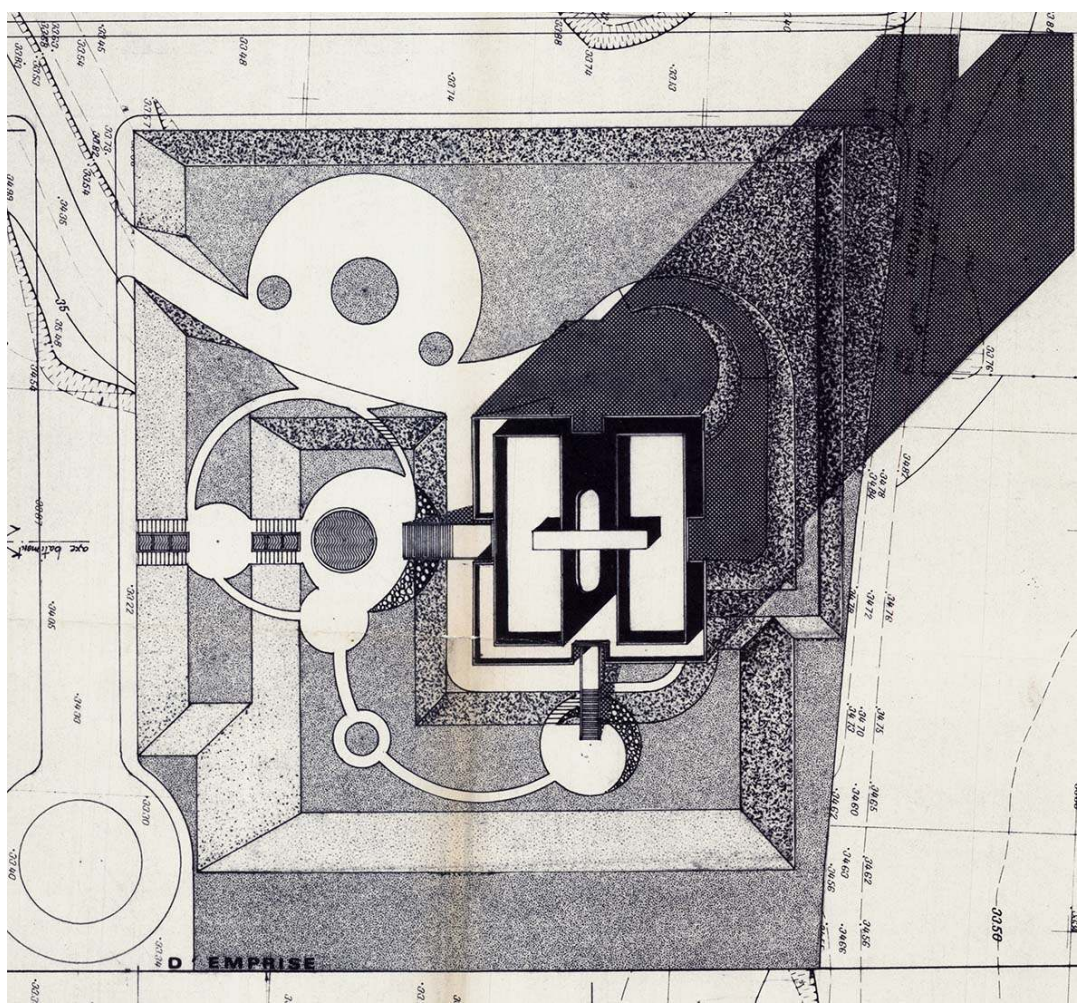

«Plan masse », projet d'appel d'offres 1971, détail. L'abandon de l'urbanisme sur dalle est acté, mais l'accès est toujours prévu depuis la voie qui longe à l'ouest la parcelle, devenue une voie en impasse. Cette voie ne sera pas réalisée et le parcours d'accès devra former un coude pour aboutir à l'actuelle rue des Archives, au nord de la parcelle. Dossier d'appel d'offres, éch. 1/500.

(c) Archives départementales du Val-de-Marne, 1525 W 28.

Les offres proposées par les entreprises dépassent l'enveloppe prévue de 9180000 francs. L'offre la "moins disante", de l'entreprise Grands Travaux en béton armé, est de 12726000 francs $^{12}$. Des modifications sont donc apportées au projet par les architectes, en accord avec la direction des Archives de France $^{13}$, mais une contribution supplémentaire de l'État et du département est néanmoins nécessaire pour assurer le financement du bâtiment.

Les modifications principales concernent la réduction de l'ambition du projet paysager, l'« allègement des façades » et la réduction des installations techniques et des surfaces de rayonnages. Cet allègement correspond à l'abandon des façades en panneaux préfabriqués des silos, qui demandaient la mise au point d'une fabrication spécifique, dont les parois seront finalement en voile béton par coffrage glissant (fig. 8). L'habillage des silos, qui était prévu en panneaux vitrés sur la base d'un calepinage régulier de panneaux rectangulaires, est remplacé par un revêtement en lames métalliques type Luxalon, produites par l'entreprise Hunter Douglas. 


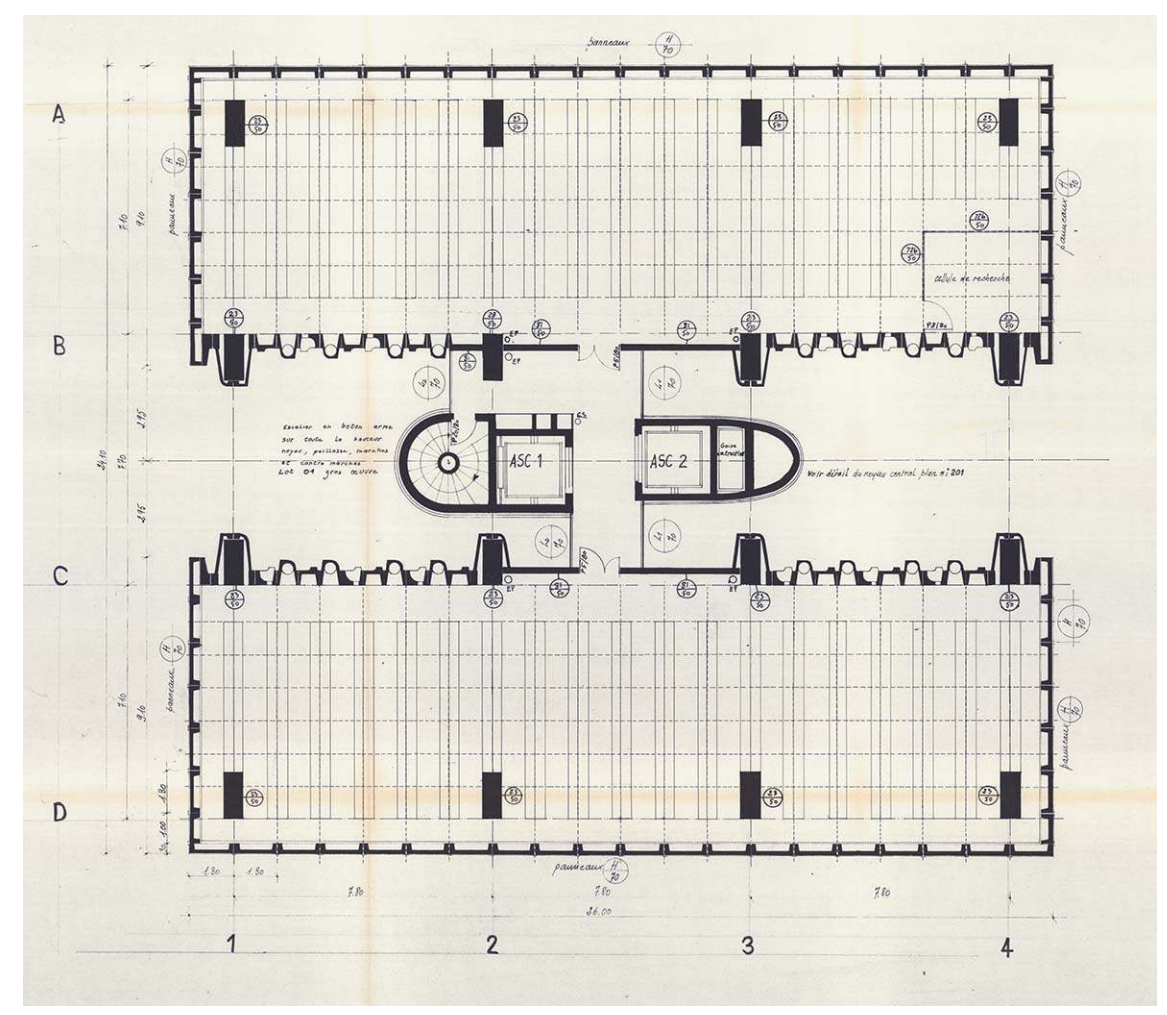

«Plan étage courant » des silos, projet d'appel d'offres 1971, détail. La solution constructive en panneaux préfabriqués et planchers intermédiaires sera remplacée par des voiles coulés dans des coffrages glissants. Dossier d'appel d'offres, éch. 1/50.

(c) Archives départementales du Val-de-Marne, 1525 W 28.

Les photos des maquettes d'étude donnent un aperçu de ces modifications (fig. 9). L'habillage prévu initialement réduisait l'impact des larges surfaces aveugles des silos et instaurait un dialogue intéressant avec l'hôtel de la préfecture, en cours d'achèvement en $1970^{14}$. Adopter la solution innovante en double vitrage isolant et réfléchissant (Thermopane Stopray) de la préfecture aurait souligné l'échelle départementale des archives, tout en générant une certaine ambiguïté quant à l'utilisation des volumes hauts. L'option finalement retenue d'un habillage en lames d'aluminium colorées, procédé encore à un stade expérimental sur des surfaces verticales extérieures aussi importantes, confère une singularité à l'édifice en affichant de manière plus claire l'affectation des silos à des réserves qui ne nécessitent pas d'ouvertures. 


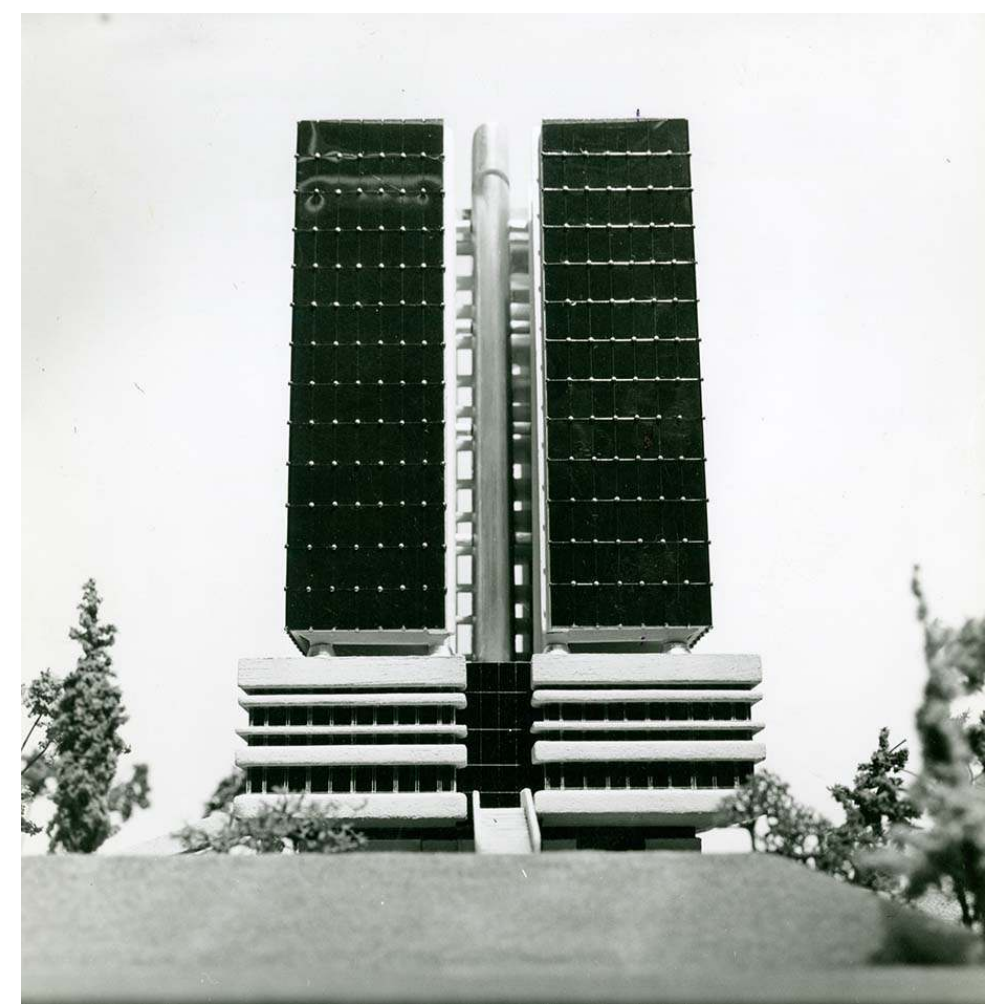

Photo de la maquette du projet d'appel d'offres, façade sud. Le revêtement en panneaux vitrés entre en résonnance avec le projet de l'hôtel de la préfecture en cours de réalisation.

Phot. Jan Coussi, date tirage 1974. (c) Archives départementales du Val-de-Marne, 1525 W 28.

L'évolution du revêtement semble avoir brouillé la communication pendant le chantier. Un article publié dans Le Courrier du Val-de-Marne est illustré par la maquette de la première version tout en décrivant un revêtement «en motifs polychromes de Luxalon dans les tons beige, brun et ocre $»^{15}$.

La maquette d'étude du projet définitif donne un aperçu assez fidèle du résultat final (fig. 10). La séparation et la superposition des fonctions sont accentuées par un habile contraste des matières. Les couches alternées d'allèges saillantes en béton désactivé et de fenêtres en bandeaux soulignent l'horizontalité du volume bas, porté par un socle en retrait qui suspend l'ensemble. Dans le vide ménagé au-dessus sont posés les poteaux foncés qui portent les silos dont le tramage accentue la verticalité. La superposition de bandeaux continus d'éléments préfabriqués en béton et de vitrages est expérimentée par l'agence au cours de ces mêmes années dans le projet de la caisse régionale du Crédit agricole de Lattes, dans l'Hérault (1974). Les effets de contraste entre des surfaces en béton et des trames métalliques ont déjà été expérimentés dans le projet de la Maison de l'agriculture à Montpellier (1969-1973) ${ }^{16}$. Le bâtiment des archives départementales à Créteil contribue à une recherche plastique et constructive pour exprimer la modernité des nouveaux équipements. 


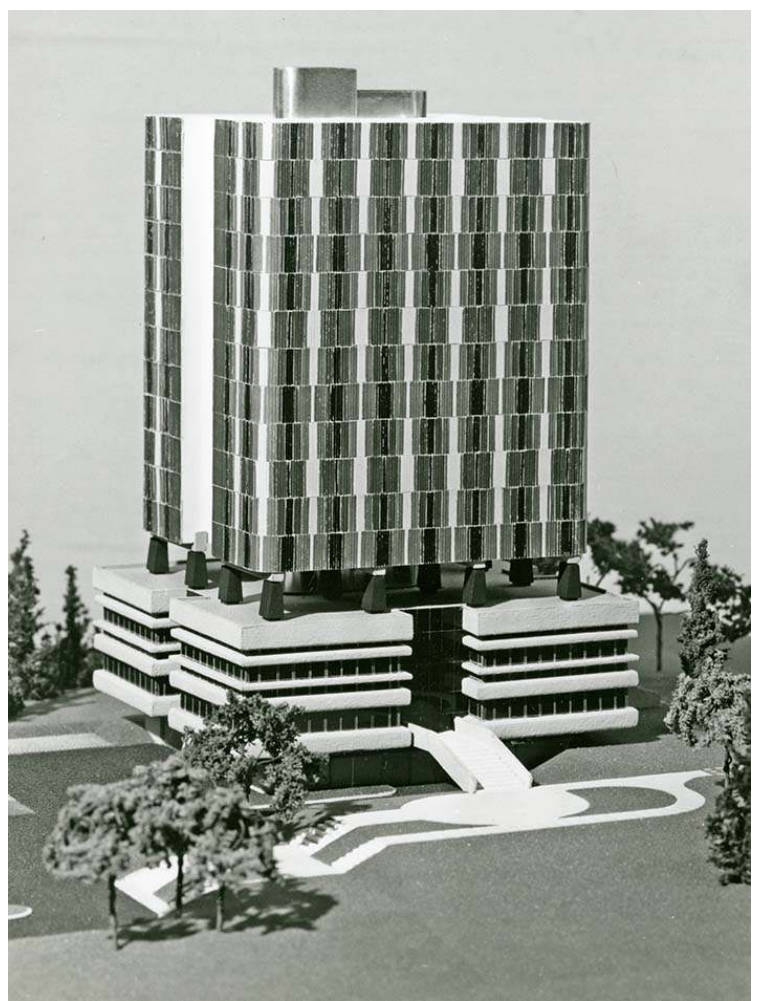

Photo de la maquette du projet définitif. La maquette met en évidence le rôle plastique de la terrasse intermédiaire et, dans le volume bas, la complémentarité entre le ton clair des bandeaux en béton et les surfaces vitrées.

Phot. Jan Coussi, date tirage 1973. @ Archives départementales du Val-de-Marne, 1525 W 28.

\section{Le déroulement du chantier et les aléas de l'expérimentation}

Selon une séquence habituelle, à la phase d'ajustement du projet pour maitriser le montant global des dépenses fait suite un chantier difficile. La correspondance fait émerger les relations conflictuelles entre les maitres d'œuvre et l'entreprise générale, la société Balency-Briard ${ }^{17}$.

Au-delà des retards dans les rendus des plans de détail, un premier conflit important éclate avec le refus des pièces préfabriquées en béton lavé destinées au volume bas, après le constat d'une homogénéité insuffisante des agrégats utilisés. Les retards accumulés aboutissent à un décalage de plusieurs semaines par rapport au planning prévisionnel ${ }^{18}$. En même temps, les joints destinés à assurer l'étanchéité entre les éléments préfabriqués se révèlent défaillants et nécessitent des reprises ponctuelles. D'autres retards sont signalés dans l'achèvement des parois des silos ou la pose des planchers autoportants du système de rayonnage Ronéo dans les réserves. L'entreprise générale est considérée responsable et le décompte des pénalités de retard démarre le $1^{\mathrm{er}}$ mai 1974 (fig. 11). 


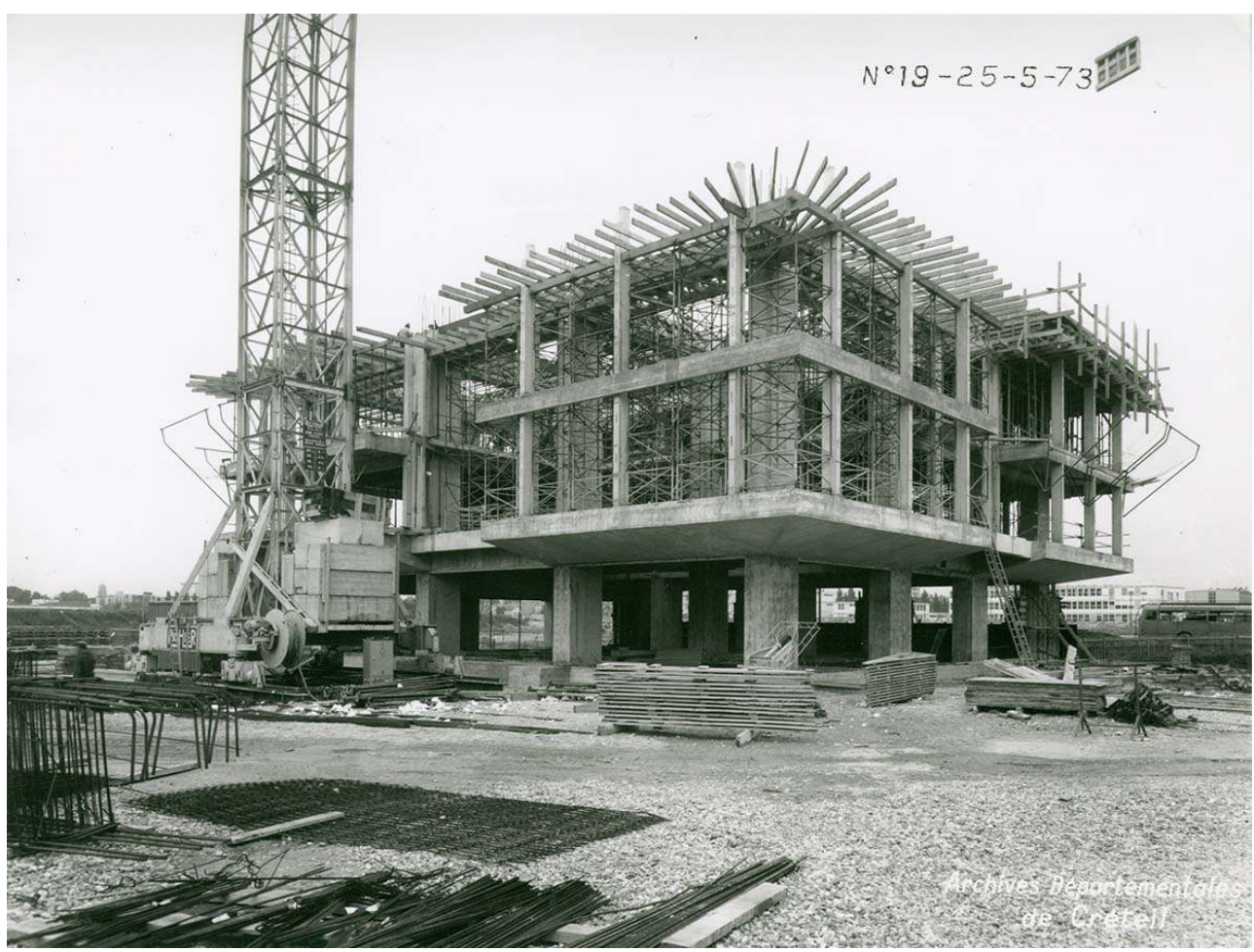

Photo de chantier pendant la réalisation des structures en béton armé. Les poteaux fondés en profondeur traversent le volume bas et portent les planchers et les volumes des silos.

Phot. H. Fernier, 1973. (C) Archives départementales du Val-de-Marne, 45 W 53.

Mais les déboires de ce chantier ne sont pas terminés. La fixation du bardage en lames métalliques pose des problèmes considérables, notamment en raison des arrondis qui raccordent les surfaces des silos. Pendant l'été 1974, avant la réception des travaux, une tempête révèle la défaillance du système d'accrochage des lames. Des défauts d'étanchéité du revêtement métallique apparaissent au cours de l'année 1975 et les architectes demandent une révision complète. La vêture mise en œuvre posant des problèmes, des essais sont demandés au Centre scientifique et technique du bâtiment. Le bâtiment des archives « semble avoir été le premier chantier réalisé par la Société Hunter Douglas qui ne bénéficiait pas alors de l'expérience acquise aujourd'hui ${ }^{19}$. Le système de fixation n'est pas adapté aux surfaces arrondies qui demanderaient la fabrication de pièces spéciales. D'autre part, pour l'ensemble des parties planes, les dispositifs d'accrochage sont insuffisants pour assurer une bonne tenue dans le temps. Suite aux problèmes apparus dans le bâtiment des archives, le fabricant Hunter Douglas fait procéder à d'autres essais pour établir un nouveau manuel de pose qui préconise des lisses plus rapprochées et l'augmentation du nombre de points de fixation (fig. 12). 


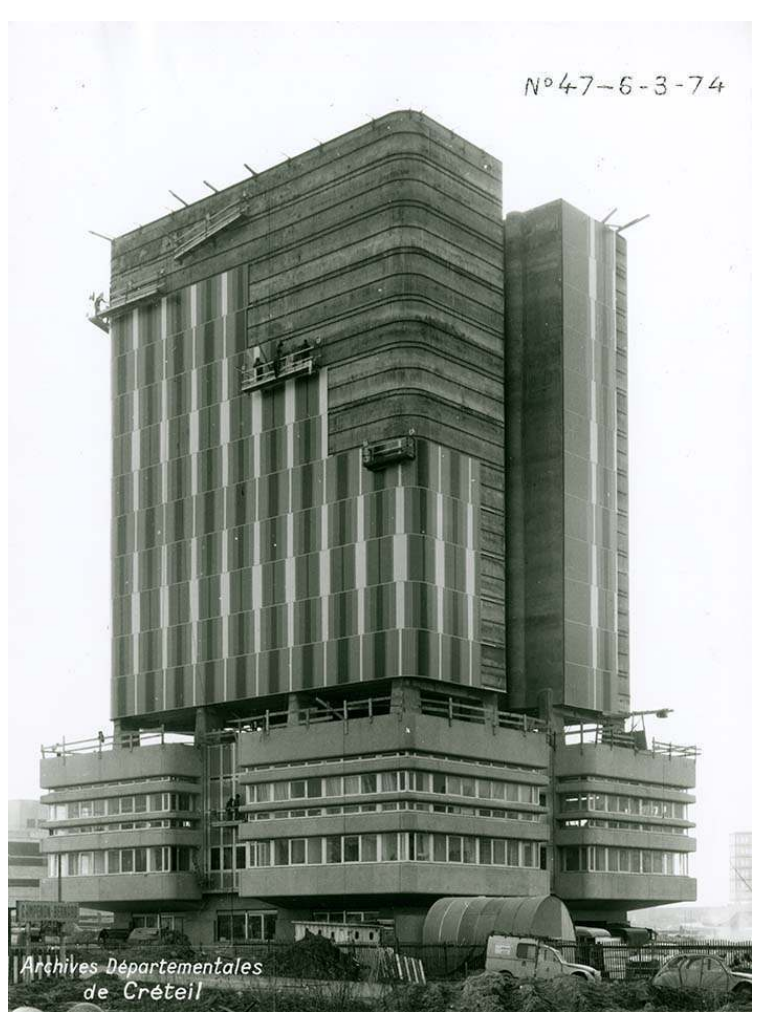

Photo de chantier pendant la pose du bardage en lames Luxalon - Hunter Douglas, 1974. Le volume bas est achevé. La pose du bardage montre les espacements importants entre les lisses qui ont engendré les défaillances du revêtement, notamment au niveau des arrondis.

(c) Archives départementales du Val-de-Marne, 45 W 53.

Les formes inédites adoptées dans le bâtiment des archives ont donc servi de prototypes pour vérifier la tenue de ce type de bardage. Si les résultats des essais assurent la pérennité du revêtement en lames Luxalon dans des nouveaux chantiers, pour le bâtiment des archives, le problème est loin d'être réglé. En 1979 et en 1981, des détachements sont à nouveau signalés et un expert est nommé par le tribunal administratif de Paris en novembre 1981 car "la chute de ces éléments présente des grands risques pour les personnes se trouvant à la périphérie du bâtiment $»^{20}$. Les architectes attribuent le choix de la solution en lames d'aluminium laqué à la société Sobea $^{21}$ qui a sous-traité la pose aux entreprises Jacqmin. Les maitres d'œuvre et l'entreprise essaient, chacun de leur côté, de réduire leur part de responsabilité. Le jugement du tribunal administratif du 2 octobre 1984 condamne, conjointement et solidairement, les architectes et l'entreprise à payer la somme de 123818 francs HT pour réaliser le remplacement nécessaire. L'entreprise qui a proposé et posé le bardage doit payer $80 \%$ de la somme. Le contentieux se poursuit jusqu'en mars 1991, avec la notification d'une décision rendue par le Conseil d'État qui redéfinit le partage des dommages à $50 \%$ entre architectes et entreprise. Finalement, l'ensemble du bardage est déposé et remplacé en 1992 en gardant le même calepinage des lames.

Ces travaux ont fait l'objet d'un reportage vidéo qui permet aujourd'hui de constater des techniques de pose inefficaces, qui expliquent notamment le faible niveau de performance de l'isolation mise en œuvre entre la paroi en béton et le bardage ${ }^{22}$. Le crédit accordé aux nouveaux matériaux et aux innovations constructives, loué dans les 
publications récentes dédiées à l'agence Badani et Roux-Dorlut, a parfois engendré des situations inconfortables dont l'histoire de l'architecture fait rarement le récit. L'appétence des constructeurs de la modernité pour l'expérimentation a été jalonnée d'erreurs et de défaillances qui nous apprennent beaucoup sur l'avancement chaotique d'un savoir-faire dont nous bénéficions largement dans la conception des constructions isolées par l'extérieur.

\section{La réception du bâtiment à lames aluminium colorées} bâtiment des archives ne passent pas inaperçues aux yeux des habitants qui commencent à s'installer. Les articles, naturellement élogieux, publiés dans Le Courrier du Val-de-Marne, évoquent le rôle urbain et l'originalité du projet. «C'est un monument qui s'élève en un quadrilatère imposant. Il est revêtu de lames métalliques en quatre teintes: champagne, noir, blanc et doré $\aleph^{23}$. D'autres s'interrogent sur la modernité foisonnante qui prend forme dans la plaine de Créteil. Un article paru dans Le Figaro littéraire en 1976 donne un aperçu du contexte de ce «palais futuriste » au milieu d'un nouveau paysage urbain qui questionne : «Est-ce-beau? Est-ce laid? La question ne se pose même pas. L'univers de Créteil est déconcertant mais fort, et le navigateur y éprouve un vif sentiment d'exaltation. »Et l'apparition du nouvel équipement est observée dans ce cadre en mutation : "Le temps possède son île à Créteil : l'immeuble des archives (...) L'ensemble évoque les grands silos de la Beauce, d'où coulent les grains. Ici, ce seront les papiers... » ${ }^{24}$.

La critique architecturale ne semble pas s'être intéressée au projet et la réception immédiate dans la presse professionnelle s'intéresse principalement à la pertinence de la réponse au programme et aux innovations constructives. Les solutions adoptées pour la réalisation des silos avec la technique du coffrage glissant sont détaillées en 1976 par J.J.Guiglio, ingénieur, directeur de travaux de l'entreprise Balency-Briard, dans Le Bâtiment-Bâtir ${ }^{25}$. Le revêtement de lames métalliques Luxalon - Hunter Douglas, appareillé "suivant un carton spécialement étudié par les architectes », est présenté en 1974 dans Le Mur vivant ${ }^{26}$. Oubliant le contentieux et les défaillances du système d'accrochage, le fabricant reproduira le bâtiment des archives dans les fiches de son catalogue.

Les photographies de l'achèvement du chantier saisissent le bâtiment dans son intégrité volumétrique et colorimétrique. La composition générale se fonde sur un jeu de contrastes : opacité/transparence, horizontalité/verticalité, arrondis/arêtes vives, béton de gravillons/lames métalliques. La clarté de la volumétrie se fonde sur une interprétation originale de la superposition des masses par le retrait du soubassement et le vide de la terrasse intermédiaire. L'utilisation habile des surfaces courbes équilibre les contrastes affichés entre les matériaux et les volumes. La capacité du duo d'architectes à sculpter des objets architecturaux est particulièrement efficace dans l'effet de tension recherché pour le traitement de la faille entre les deux silos où la séparation des parois à cannelures inversées semble forcée par le noyau des circulations verticales. Ces aspects continuent à qualifier le bâtiment malgré les modifications ponctuelles liées aux mises aux normes et à l'adaptation aux nouveaux besoins.

29 Le bâtiment des archives est présenté dans le récent ouvrage consacré à l'œuvre de Badani et Roux-Dorlut ${ }^{27}$ à l'intérieur du chapitre consacré aux interventions à échelle urbaine car il participe à une contribution plus large de l'agence dans la définition du 
paysage du Nouveau Créteil. Cependant, la densification de son environnement proche et l'extrême variété des architectures qui caractérise celui-ci ont affaibli l'impact des deux volumes superposés, dont l'agencement n'est perceptible dans son ensemble que depuis quelques points de vue privilégiés (fig. 13, fig. 14). Le bâtiment peut être assez bien repéré depuis les rues environnantes mais son rôle urbain est réduit par l'enclavement de la rue des Archives et l'incongruité des parcours d'accès piétons. Le public arrivant de la station de métro doit emprunter un passage dissimulé et aboutir au portail d'accès à la parcelle depuis lequel la porte du bâtiment n'est pas visible.

Figure 13

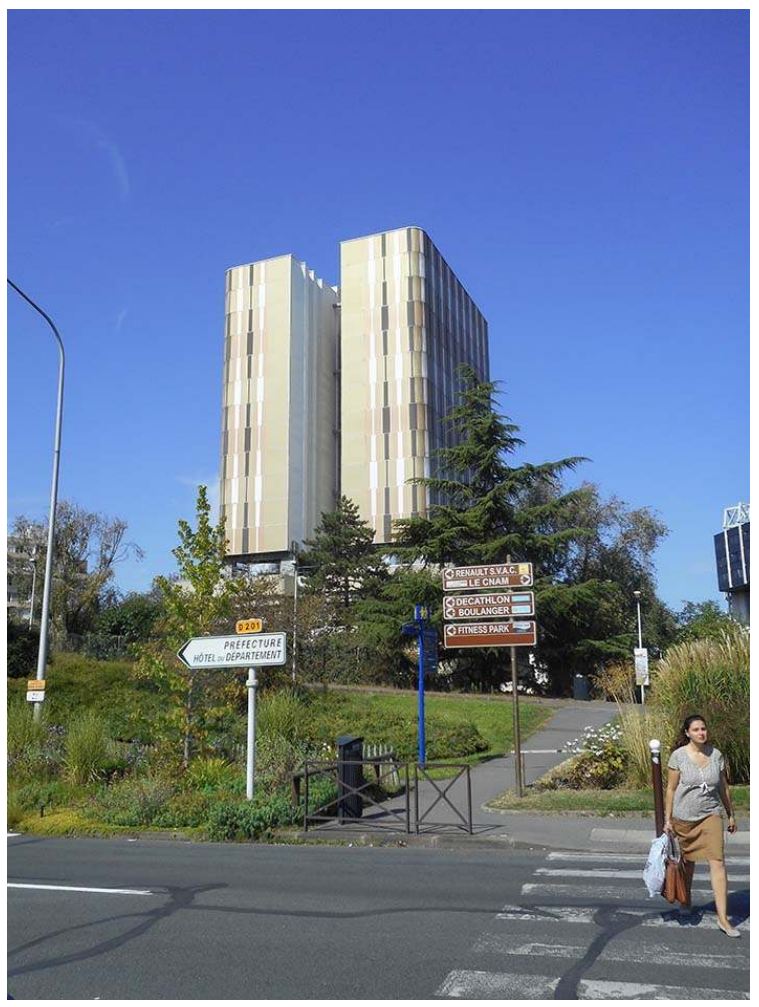

Vue des archives départementales depuis la traversée piétonne empruntée par la majorité des visiteurs. Le volume bas est masqué par les plantations et les installations techniques occupent le vide de la terrasse intermédiaire.

Phot. Franca Malservisi. @ Franca Malservisi. 


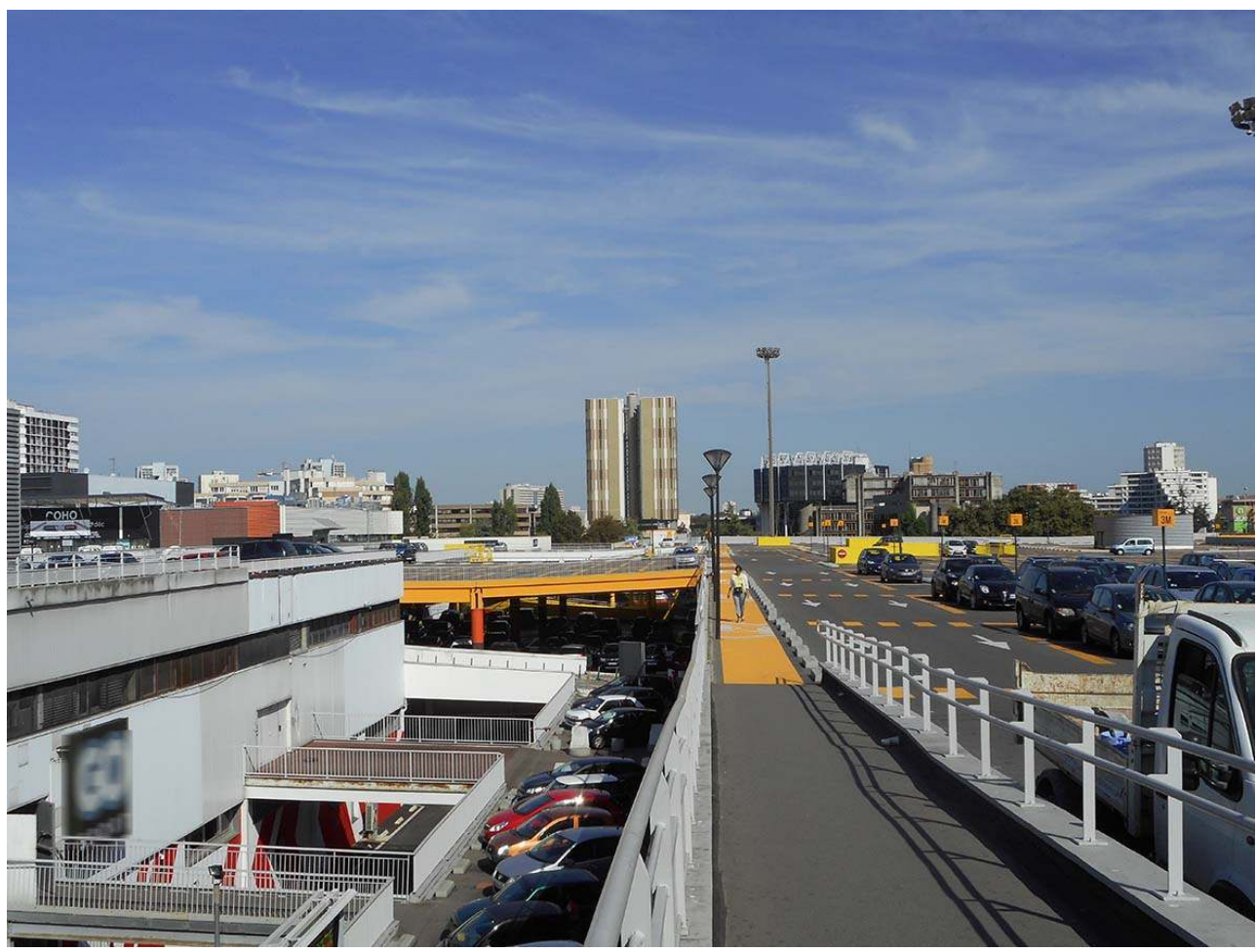

Perception de la faille entre les silos depuis le passage d'entrée au centre commercial Créteil Soleil.

Phot. Franca Malservisi. (c) Franca Malservisi.

Les études sur l'histoire urbaine du Nouveau Créteil et l'intérêt récent pour l'œuvre de l'agence Badani et Roux-Dorlut sont une première étape vers des travaux critiques sur la cohérence de leur intervention dans la ville ou sur la spécificité de la modernité architecturale qui s'est exprimée dans la fabrication de cet étonnant paysage urbain. L'actualité pressante des réflexions sur l'entretien et la rénovation des bâtiments impose aujourd'hui une évaluation approfondie des qualités architecturales et constructives.

\section{Les archives départementales du Val-de-Marne dans l'évolution d'une typologie d'équipement}

S'il s'inscrit pleinement dans une démarche de normalisation de la construction de nouveaux dépôts d'archives départementales, encouragée depuis 1945 par la direction des Archives de France, le bâtiment de Créteil se singularise par la qualité architecturale de l'édifice, très éloignée de la répétitivité qui caractérise souvent ceux construits pendant les Trente Glorieuses, où les faux jumeaux ne sont pas rares: Alençon, Draguignan et Mâcon, ou encore Limoges et Tulle.

En 1986, la direction des Archives de France publie un ouvrage entièrement consacré aux bâtiments d'archives aménagés entre 1965 et $1985^{28}$. Les fiches par réalisation offrent un aperçu des équipements réalisés dans les trois nouveaux départements issus du découpage administratif de l'ancien département de la Seine. Le bâtiment des architectes Badani et Roux-Dorlut est le premier réalisé et propose une solution à silos aveugles revêtus d'un bardage métallique qui sera reprise dans les bâtiments des Hauts-de-Seine 
(Nanterre) et de Seine-Saint-Denis (Bobigny), projetés par le cabinet Feypell et Zoltowski. La filiation est évidente et montre la pertinence de ces volumes aveugles aux angles arrondis séparés par des failles profondes accueillant les hauts cylindres des circulations verticales.

La présentation introductive de l'ouvrage permet de situer le projet des archives du Valde-Marne dans un moment de transition des normes de conservation et de sécurité incendie. Le système d'archivage auto-porteur retenu avait profité d'une large diffusion au cours des années 1960 et avait permis de réaliser à un coût relativement limité des hautes tours de réserves. Les incendies survenus dans les équipements publics, comme l'incendie du collège Pailleron à Paris en 1973, avaient également imposé de nouvelles normes de sécurité. Le système auto-porteur Ronéo encore en place à Créteil constitue une des toutes dernières mises en œuvre de ces rayonnages superposés à structure métallique (fig. 15).

Figure 15

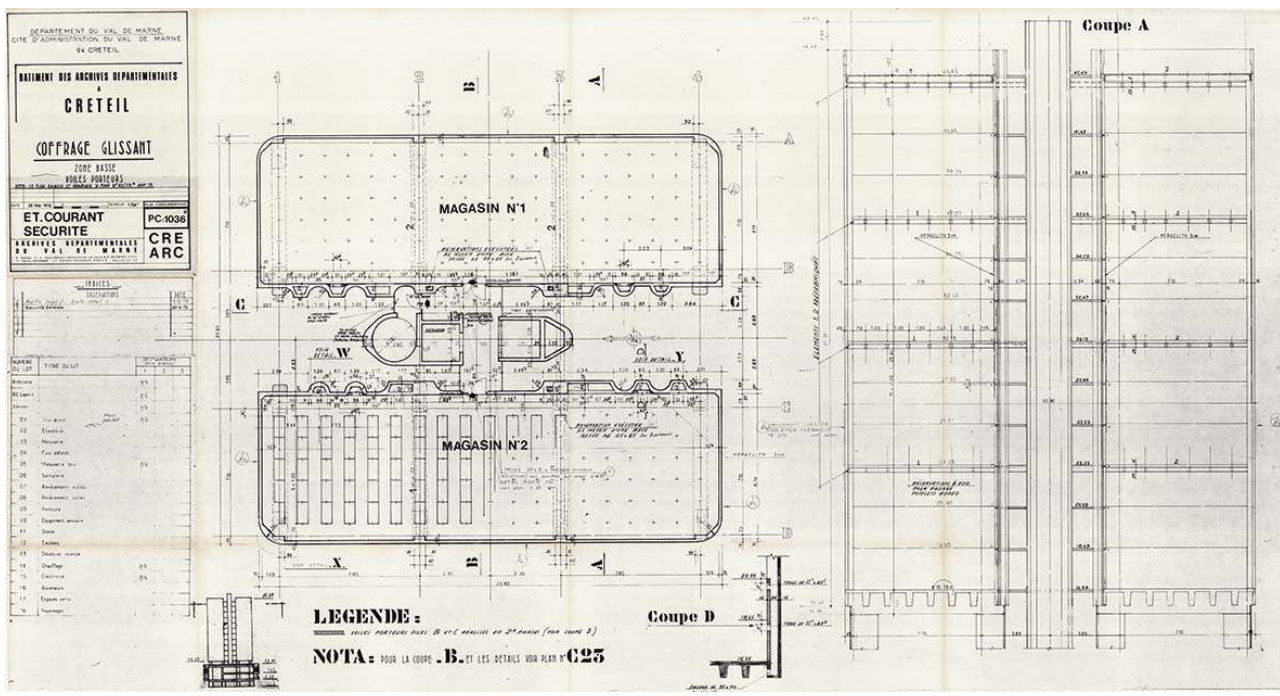

«Plan complémentaire » d'un niveau des silos établi par l'entreprise Courant-Sécurité sur un plan de détail de gros œuvre (coffrage glissant), 1974, éch. 1/50. Le plan montre les vides aménagés pour sculpter les parois qui forment la faille entre les silos.

(c) Archives départementales du Val-de-Marne, 1525 W 29. de France précise: "Conçu il y a presque quinze ans, le bâtiment ne possède pas de dispositif de maintien de la température et du degré hygrométrique dans les magasins qui ne sont pas chauffés; une ventilation forcée a été installée après-coup ${ }^{29}$. " Les deux bâtiments construits entre 1977 et 1983 à Nanterre et à Bobigny présentaient depuis leur achèvement des équipements techniques performants (ventilation des magasins, dispositifs de maintien de la température et du degré hygrométrique) qui répondaient à de nouvelles exigences pour assurer la conservation des documents.

L'étude de l'évolution du projet et de sa concrétisation permet d'attribuer au moins partiellement les défaillances dans le contrôle de la température et de l'hygrométrie aux réductions des prestations lors de l'appel d'offres. En effet, ces modifications comprenaient la réduction du rayonnage à une première tranche de 8000 mètres linéaires et celle des équipements techniques dans les parties des silos sans rayonnages. 
Lors de leur achèvement, les silos ne comportent que trois niveaux d'archives et ce n'est que progressivement que les volumes vides seront remplis avec des extensions des surfaces de stockage. L'adaptation des rayonnages à l'accumulation des fonds a vraisemblablement engendré une adaptation des équipements techniques au cas par cas, manquant de cohérence, responsable d'une partie des dysfonctionnements constatés (fig. 16).

Figure 16

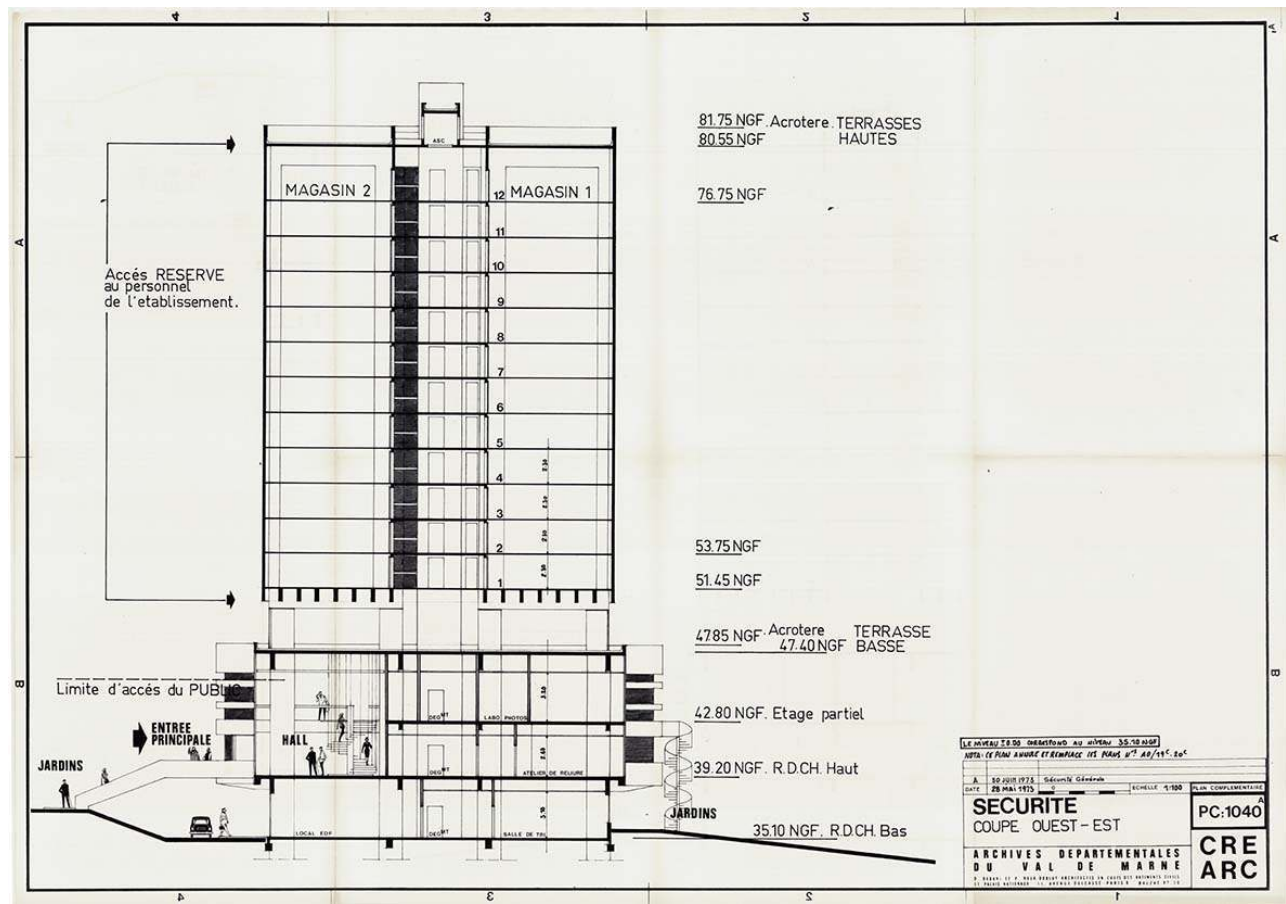

" Coupe ouest-est. Sécurité », coupe montrant le fonctionnement du bâtiment, éch. 1/50, 1973. Les planchers autoportants des réserves seront réalisés par tranches successives.

(c) Archives départementales du Val-de-Marne, 1525 W 29.

Une partie des travaux réalisés pour adapter le site aux nouveaux besoins et améliorer les installations techniques ont affecté l'aménagement des espaces libres et l'aspect extérieur du bâtiment. L'adaptation des installations de chauffage et de climatisation a comporté la mise en œuvre de volumes techniques imposants dans le vide de la terrasse intermédiaire et dans les espaces verts plantés. Le remplacement des éléments de bardage a engendré la réduction de l'épaisseur $d u$ bandeau qui marquait l'arête vive de l'acrotère. Les contraintes de sécurité ont imposé la mise en place d'un garde-corps au niveau de la terrasse basse. La fixation de cet élément à l'acrotère, pour permettre d'intervenir ultérieurement sur l'étanchéité, en augmente l'impact visuel. Au cours des travaux de réhabilitation du volume bas, terminés en 2012, les menuiseries en aluminium anodisé d'origine ont été remplacées par des menuiseries double vitrage en aluminium laqué blanc. Ce choix a réduit la continuité des surfaces vitrées et provoqué une incohérence avec les murs rideaux situés entre les silos. Lors de cette dernière campagne de travaux, une extension est venue compléter l'équipement. Le positionnement de ce nouveau volume entérine définitivement l'abandon d'un parcours d'accès depuis l'ouest en se positionnant en face de l'entrée principale. 


\section{Conclusion}

La permanence de l'affectation initiale et l'entretien régulier des services techniques du conseil départemental du Val-de-Marne ont assuré un bon état de conservation des dispositions initiales, malgré les modifications ponctuelles signalées (fig. 17). Une première étude commandée par le conseil départemental en 2014 et le pré-diagnostic énergétique, réalisé conjointement à l'analyse historique et critique du bâtiment, ont permis de préciser les principales pathologies, les dispositions non conformes et les difficultés de régulation des températures et d'hygrométrie. L'évaluation du projet des archives et de son histoire constructive a défini des prescriptions à respecter dans le cadre d'une rénovation du bâtiment. Les échanges avec les BET et les fournisseurs ont permis de vérifier que le remplacement du bardage métallique Hunter Douglas est envisageable sans modification du principe de calepinage existant, car les lames et les tonalités sont toujours disponibles. Une expérimentation risquée a abouti à une solution technique qui peut aujourd'hui faciliter l'amélioration énergétique mais sa rapide obsolescence est problématique pour la durée des vêtures métalliques, largement présentes dans les projets contemporains. L'opération reste délicate pour dessiner dans le détail les éléments de raccord nécessaires pour absorber la surépaisseur d'un isolant performant.

\section{Figure 17}

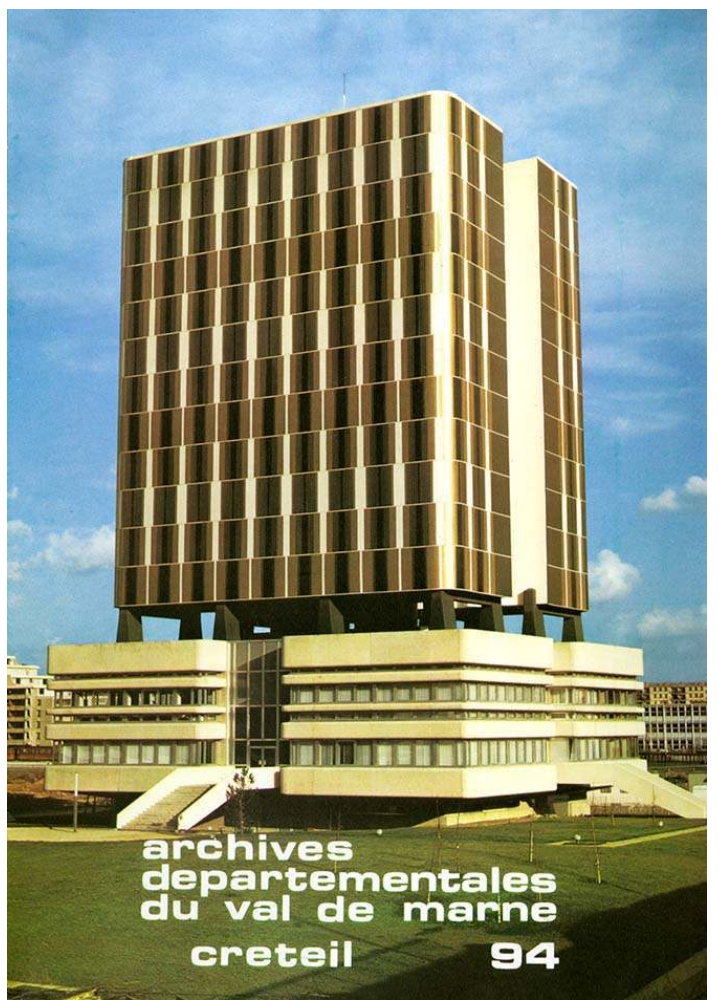

Couverture de la plaquette imprimée lors de l'inauguration du bâtiment, le 16 janvier 1975.

L'équipement émerge dans un environnement encore libre de constructions. Les escaliers d'accès se posent sur les talus artificiels qui rehaussent la parcelle au sud et à l'ouest.

(c) Archives départementales du Val-de-Marne, 1525 W 39. 
38

principe d'une réflexion globale a donc été retenu pour que le projet à venir se base sur une connaissance approfondie d'un équipement qui garde les traces d'une histoire constructive riche d'enseignements. Ces chantiers, fondés sur la prise en compte de l'histoire urbaine du site et de la cohérence du projet des architectes Badani et RouxDorlut, peuvent être l'occasion d'estomper l'impact des ajouts qui nuisent à la perception de l'ensemble, de repenser la relation entre le bâtiment et son environnement proche, de valoriser sa visibilité dans le paysage jusqu'à une éventuelle reconfiguration des parcours d'accès. Trace matérielle de la remise en question de l'urbanisme sur dalle dans la construction du Nouveau Créteil, témoin d'un épisode de l'introduction des habillages métalliques en façade, exemple significatif des recherches architecturales d'une agence prolifique active au cours de la deuxième moitié $\mathrm{du} \mathrm{xx}^{\mathrm{e}}$ siècle, le bâtiment des archives départementales du Val-de-Marne à Créteil mérite l'attention du conseil départemental pour en assurer la pérennité.

\section{NOTES}

1. - L'étude globale a été établie par le conseil d'architecture, de l'urbanisme et de l'environnement (CAUE Val-de-Marne) et les ingénieurs-conseils de l'agence de l'énergie du Valde-Marne en février 2017. Pour une présentation du contexte du projet des archives départementales, voir dans ce numéro : CORCUFF, Marie-Andrée. « Les Archives départementales du Val-de-Marne : un bâtiment structurant de l'administration du nouveau département ", In Situ [En ligne], 34 2018 , mis en ligne le 17 juillet 2018, consulté le 17 juillet 2018. URL: http:// journals.openedition.org/insitu/1607.

2. - MALSERVISI, Franca. Archives départementales du Val-de-Marne, diagnostic patrimonial. CAUE Val-de-Marne, direction des archives départementales, direction des bâtiments, décembre 2016.

3. - Voir les articles concernant les récentes rénovations de bâtiments de l'agence et les publications citées dans les notes iv et v: VÉRAN, Cyrille. "Dominique Perrault. Tours de bureaux, Boulogne-Billancourt ». AMC, $\mathrm{n}^{\circ}$ 248, février 2016, p. 24-31; GUISLAIN, Margot. « Béton préfabriqué ». $A M C, \mathrm{n}^{\circ} 242$, mai 2015, p. $75-86$; "Citylights, tête de pont du Grand Paris ». La Pierre d'angle, $\mathrm{n}^{\circ}$ 60, octobre 2012, p. 16-17; SÉRON-PIERRE, Catherine. " La nouvelle image du Forum à Boulogne-Billancourt. Architectes Eliet \& Lehman ». AMC, $\mathrm{n}^{\circ} 217$, septembre 2012, p. 24 ; REDECKE, Sebastian. « Boulogne-Billancourt ». Bauwelt, $\mathrm{n}^{\circ}$ 27/28, juillet 2011, p. 12-45.

4. - DELHUMEAU, Gwenaël, LEMONIER, Aurélien. Badani, Roux-Dorlut architectes. La conquête du milieu. Orléans : Éditions HYX, 2016.

5. - LEMPEREUR, Hubert. «Badani \& Roux-Dorlut. Des orfèvres de la grande échelle ». AMC, $\mathrm{n}^{\circ}$ 224, mai 2013, p. 77-86.

6. - COUDROY de LILLE, Laurent. Une nouvelle ville: une histoire de l'urbanisme du Nouveau Créteil. Créteil : Institut d'urbanisme de Paris, 2005.

7. - Note au sujet de la construction du bâtiment des archives du département du Val-de-Marne à Créteil. Préfecture du Val-de-Marne, 18 avril 1972 (AD Val-de-Marne, 1525 W 27).

8. - PV de la séance du 29 mai 1969, conseil général des Bâtiments de France (AD Val-de-Marne, 1525 W 27, p. 2).

9. - Ibid., note 6, p. 3 . 
10. - Des silos pour abriter les magasins apparaissent par exemple aux archives départementales de l'Orne, de la Saône-et-Loire, du Var, du Pas-de-Calais. (HOTTIN, Christian. «Conservation préventive et bâtiments d'archives ». 2001, voir sur le site : https://halshs.archives-ouvertes.fr/ halshs-00087949.

11. - Rapport de présentation. Architectes Badani et Roux-Dorlut, 15 mai 1910 (AD Val-de-Marne, 1525 W 27).

12. - Voir la conclusion du rapport de la commission départementale d'analyse des réponses du 18 décembre 1971 (AD Val-de-Marne, 1525 W 27).

13. - Voir la note de la préfecture du Val-de-Marne, 18 avril 1972 (AD Val-de-Marne, 1525 W 27), qui liste les modifications : allègements de la façade; suppression de la clôture, des bassins extérieurs et simplification des plantations et des travaux de voirie et réseaux divers (VRD) ; suppression de la chambre forte; réduction du rayonnage à une première tranche de 8000 mètres linéaires et réduction des équipements dans les parties sans rayonnages; remplacement de certains matériaux de revêtement; suppression de l'incinérateur et de sa cheminée; suppression de deux groupes électrogènes de secours et de l'éclairage de secours; suppression d'un ascenseur sur deux; suppression des courants faibles et de l'antenne collective de radio télévision.

14. - Après le déménagement du service dans le nouveau bâtiment, Le Courrier du Val-de-Marne du 21 août 1974 revient sur le projet en donnant la parole à l'un des architectes de l'agence Badani et Roux-Dorlut. «L'architecte Dorent (...) avait prévu des plaques de verre coloré comme la préfecture, mais ornées de gros clous d'aluminium. Cette solution s'étant révélée trop onéreuse, le projet a été transformé. » «Le nouveau bâtiment des Archives du Val-de-Marne sera inauguré en janvier ». Le Courrier du Val-de-Marne, octobre 1974, $\mathrm{n}^{\circ} 203$, p. 6.

15. - «La maquette des futures archives départementales». Le Courrier du Val-de-Marne, octobre 1973, n 161 , p. 10.

16. - DELHUMEAU, Gwenaël, LEMONIER, Aurélien. Op. cit., p. 106-110.

17. - Voir la correspondance relative au déroulement du chantier de construction et les séries de plans conservés aux archives départementales (AD Val-de-Marne, $1525 \mathrm{~W}$ 28-41).

18. - Le 31 octobre 1973, dans le compte rendu $\mathrm{n}^{\circ} 42$, il est constaté un retard de 10 semaines sur la pose des panneaux (AD Val-de-Marne, $1525 \mathrm{~W} 29$ ).

19. - Courrier du 9 février 1979 adressé aux architectes Badani et Roux-Dorlut par le Centre scientifique et technique du bâtiment, qui valide les nouveaux abaques de pose de l'entreprise Hunter Douglas (AD Val-de-Marne, 1525 W 39).

20. - Rapport de l'expert Jean Monnet arch. DPLG, tribunal administratif de Paris, RA du 24 novembre 1981 (AD Val-de-Marne, 1525 W 39).

21. - Dans un phénomène général de regroupement des entreprises du bâtiment, Balency-Briard fait partie en 1981 de l'entreprise Sobea, qui fusionnera avec le SGE en devenant une filiale de Vinci.

22. - « Réfection totale du bardage extérieur du bâtiment des Archives départementales du Valde-Marne ", réalisation et musique Jean-Jacques GUILLAUME, production Conseil général du Valde-Marne, 1992 (AD Val-de-Marne, 21AV/116).

23. - «M. Michel Guy inaugure les Archives départementales». Le Courrier du Val-de-Marne, 30 janvier $1975, n^{\circ} 226$, p. 5 .

24. - PRASTEAU, Jean. «Un archipel de papiers à Créteil ». Le Figaro littéraire, 11 septembre 1976, $\mathrm{n}^{\circ} 3,1582$, p. II.

25. - «Les archives départementales du Val-de-Marne». Le Bâtiment - Bâtir, nº 4, avril 1976, p. 63-64.

26. - « Lamellé polychrome ». Le Mur vivant, 1974, $\mathrm{n}^{\circ} 34$, p. 15-16.

27. - Ibid., note iv, p. 135-165. 
28. - Direction des Archives de France. Bâtiments d'archives. Vingt ans d'architecture française, 1965-1985. Paris : Archives nationales, 1986, p. 202.

29. - Ibid., note xxviii, p. 28-29.

\section{RÉSUMÉS}

Trois nouveaux équipements constituent la contribution essentielle des architectes Badani et Roux-Dorlut au paysage du Nouveau Créteil : l'hôtel de la préfecture, le palais de justice et le bâtiment des archives départementales. La connaissance du processus qui aboutit à la réalisation de ces édifices au début des années 1970 s'avère aujourd'hui nécessaire pour en assurer la pérennité et répondre à une évolution des besoins. L'étude historique du bâtiment des archives, destinée à nourrir une réflexion globale sur l'amélioration des performances thermiques, permet un regard rétrospectif sur les nombreux aspects constructifs et formels qui caractérisent les expressions architecturales de cette modernité confiante. Les phases de conception du projet confirment l'inventivité de la recherche plastique menée par ce duo d'architectes et les liens étroits entre les trois équipements cristoliens. Le projet du bâtiment des archives explore une séparation des fonctions avec un volume bas, affecté aux locaux administratifs et à l'accueil du public, sur lequel sont posés les deux silos destinés aux réserves. Grace à un habile jeu de contrastes entre la souplesse des arrondis et les arêtes vives, entre béton lavé et murs rideaux, entre volumes aveugles et failles transparentes, le bâtiment exprime une recherche qui surprend dans un équipement aux dimensions relativement modestes. L'évolution du projet éclaire certains aspects des défaillances techniques constatées. En cours de chantier, les transformations qui touchent les aménagements urbains et l'environnement proche apparaissent comme les raisons principales d'une relative faiblesse de la relation entre le bâtiment et l'espace public. Enfin, l'histoire conflictuelle de la mise en œuvre du bardage en lames aluminium Luxalon offre un aperçu des aléas inhérents à l'adoption de nouveaux matériaux et nouvelles techniques. À travers une compréhension des interactions entre innovation formelle et expérimentation constructive se dégagent ainsi des pistes pour assurer l'entretien et la valorisation du bâtiment.

Three modern public facilities constitute an essential contribution by the architects Badani and Roux-Dorlut to the landscape of the New Creteil: the hotel of the prefecture, the courthouse and the building of the departmental archives. Information about the construction of these buildings in the early 1970s is now necessary to ensure their sustainability and to respond to their evolving needs. An historical survey of the archives building, originally part of a global reflection on the improvement of thermal performance, allows for a retrospective look at many constructive and formal aspects typical of such architectural expressions of modernity. The design phase of the project confirms the inventiveness of the architectural duo's research into sculptural forms and the close links between these three structures at Créteil. The archives building project explores a functional organisation that separates a low concrete volume, for the administrative offices and the reception of the public, and the two silos for archive storage. Thanks to the intelligent use of contrast between aesthetics such as rounded versus sharp edges, deactivated concrete versus curtain walls, and blind volumes versus transparent splitting, the building keeps us searching and hoping for more, which is surprising for a public facility of such modest dimensions. The evolution of the project also explains some aspects of the technical problems observed. The transformation of urban development during construction and the evolution of the surrounding 
environment appear as the main reasons for the relatively weak relationship between the building and its surrounding public space. Finally, the conflicting history of Luxalon aluminium strip cladding provides an overview of the hazards inherent in the adoption of new materials and techniques. Through the understanding of the interactions between formal innovation and constructive experimentation, emanate the recommendations that ensure the maintenance and enhancement of this architectural heritage.

\section{INDEX}

Keywords : innovation, construction history, construction techniques constructive, technical history, twentieth-century heritage, Créteil, Daniel Badani, Pierre Roux-Dorlut, modern architecture, departmental archives, equipment, reinforced concrete, urban project, diagnostic Mots-clés : innovation, histoire constructive, technique constructive, histoire des techniques, patrimoine du xxe siècle, Créteil, Daniel Badani, Pierre Roux-Dorlut, architecture moderne, archives départementales, équipement, béton armé, projet urbain, diagnostic

\section{AUTEUR}

\section{FRANCA MALSERVISI}

Architecte, chercheur en histoire de l'architecture f.malservisi@caue94.fr 